\title{
Preparation and Characterization of Graphene Oxide
}

\author{
Jianguo Song, ${ }^{1}$ Xinzhi Wang, ${ }^{1}$ and Chang-Tang Chang ${ }^{2}$ \\ ${ }^{1}$ College of Environment and Materials Engineering, Yantai University, 30 Qingquan Road, Laishan District, Yantai 264005, China \\ ${ }^{2}$ Department of Environmental Engineering, Ilan University, No. 1, Section 1 Shen-Lung Road, Yilan City 26047, Taiwan
}

Correspondence should be addressed to Jianguo Song; songjianguo@126.com and Chang-Tang Chang; ctchang@niu.edu.tw

Received 11 December 2013; Accepted 9 February 2014; Published 11 March 2014

Academic Editor: Wen Lei

Copyright (C) 2014 Jianguo Song et al. This is an open access article distributed under the Creative Commons Attribution License, which permits unrestricted use, distribution, and reproduction in any medium, provided the original work is properly cited.

\begin{abstract}
Graphene oxide (GO) films with two-dimensional structure were successfully prepared via the modified Hummer method. It is proven that redox method is a promising way to synthesize GO films on a large scale. Comprehensive characterizations of the properties of GO films were conducted. TEM and DFM analyses showed that GO sheets prepared in this study had single and double lamellar layer structure and a thickness of $2 \sim 3 \mathrm{~nm}$. X-ray diffraction (XRD) was selected to measure the crystal structure of GO sheet. Fourier-transform infrared spectra analyzer (FT-IR) was used to certify the presence of oxygen-containing functional groups in GO films. The tests of UV-VIS spectrometer and TGA analyzer indicated that GO sheet possessed excellent optical response and outstanding thermal stability. Elemental analyzer (EA) and X-ray photoelectron spectroscope (XPS) analyzed the components synthetic material. Simultaneously, chemical structure of GO sheet was described in this study. Discussion and references for further research on graphene are provided.
\end{abstract}

\section{Introduction}

As early as 1947, Wallace [1] first proposed the concept of graphene and studied the electronic properties of graphene using tight-binding model. It is concluded that graphite is a semiconductor without activation energy, because of the small portion of valence band of graphite extended to the conduction band, in which data established a foundation for the use of the physical properties of graphite. However, since several decades, the study of graphene is still just staying at the level of theory [2-5].

Until 2004, Novoselov and Geim [6] in the University of Manchester firstly separated the single graphene from graphite through simple mechanical peeling method. Graphene was increasing more and more attention in various areas of science and technology due to its remarkable physicochemical properties.

As a one-atom-thick, two-dimensional crystal, graphene has been considered as basic building block for all sp2 graphitic materials including fullerenes, carbon nanotubes, and graphite [6]. Owing to the special two-dimensional structure, graphene possessed many unique properties different than carbon materials, including a high specific surface area (theoretically $2630 \mathrm{~m}^{2} / \mathrm{g}$ for single-layer graphene)
[7], extraordinary electronic properties and electron transport capabilities [8-10], and high thermal conductivity $\left(\approx 5000 \mathrm{~W} \mathrm{~m}^{-1} \mathrm{~K}^{-1}\right)[11,12]$.

Currently, many methods had been explored to prepare graphene. Novoselov and Geim [6] firstly observed a single layer of graphene from highly oriented pyrolytic graphite using micromechanical exfoliation method. It is a simple way to prepare graphene, but the yield of graphene was very low, and you cannot achieve high-quality industrial production. Srivastava et al. [13] prepared "petal" graphite sheet with $20 \mathrm{~nm}$ thickness by chemical vapor deposition (CVD). Even though thickness of graphite sheet prepared by CVD method significantly decreased than ever, more impurities Ni element in graphite sheet was another new problem. Solvent stripping method [14] is a new approach that emerged in recent years. Its principle is destroying Van Der Waals Force between the graphite layers in a solvent to obtain graphene sheets. Method of solvent stripping did not destroy the structure of the graphene and presented no surface defects in the graphene sheet. However, the low yield of graphene was still. Redox method [15-21] is the most popular method to prepare graphene and graphite oxide. During the oxidation process, graphite crystal was treated with strong oxidizing 
agents and carried oxygen-containing functional groups into graphite oxide sheet. After thermal or ultrasonic treatment, the oxygen-containing functional groups were removed and obtained the graphene sheet. Production of graphene can be very high using redox method, and introduction of functional groups in the oxidation process provided advantages of combining the compound to improve the compatibility of matrix composite. Therefore, redox method was suitable for the preparation of graphene-based composite materials. Finally, chemical structure of GO sheet was also described in this study.

In summary, in order to turn graphene applications into reality, one must fabricate the material. The redox method is considered to be a very promising way to prepare graphene, and graphite oxide (GO), as intermediate product of preparation process has a crucial influence on the quality of graphene. Therefore, exploration for properties of graphite oxide is essential and imminent.

In view of these issues, this study successfully synthesized graphene oxide (GO) by the modified Hummers method, as the precursor for preparing graphene. The microscopic morphologies of GO sheets were characterized by transmission electron microscopy (TEM) and dynamic force microscope (DFM). The structures of the GO sheets were measured by Fourier-transform infrared spectra analyzer (FT-IR) and Xray diffraction (XRD). A UV spectrometer (UV-VIS) was used to measure the optical absorption properties of the GO sheet. Thermal stability of GO sheets was determined through the thermal gravimetric analyzer (TGA). An elemental analyzer (EA) was used to analyze the elements in the GO sheet, including $\mathrm{C}$ and $\mathrm{H}$.

\section{Experiment and Methods}

2.1. Chemicals and Materials. Graphite flake (natural, -325 mesh) with a purity of $99.8 \%$ was purchased from Alfa Aesar. All chemicals were of analytical grade and used as received.

2.2. Synthesis of Graphene Oxide (GO). GO was prepared according to the modified Hummer method [18, 22]. In detail, $5 \mathrm{~g}$ of graphite and $2.5 \mathrm{~g}$ of $\mathrm{NaNO}_{3}$ were mixed with $108 \mathrm{~mL} \mathrm{H}_{2} \mathrm{SO}_{4}$ and $12 \mathrm{~mL} \mathrm{H}_{3} \mathrm{PO}_{4}$ and stirred in an ice bath for $10 \mathrm{~min}$. Next, $15 \mathrm{~g}$ of $\mathrm{KMnO}_{4}$ were slowly added so that the temperature of the mixture remained below $5^{\circ} \mathrm{C}$. The suspension was then reacted for $2 \mathrm{~h}$ in an ice bath and stirred for $60 \mathrm{~min}$ before again being stirred in a $40^{\circ} \mathrm{C}$ water bath for $60 \mathrm{~min}$. The temperature of the mixture was adjusted to a constant $98^{\circ} \mathrm{C}$ for $60 \mathrm{~min}$ while water was added continuously. Deionized water was further added so that the volume of the suspension was $400 \mathrm{~mL}$. $15 \mathrm{~mL}$ of $\mathrm{H}_{2} \mathrm{O}_{2}$ was added after $5 \mathrm{~min}$. The reaction product was centrifuged and washed with deionized water and $5 \% \mathrm{HCl}$ solution repeatedly. Finally, the product was dried at $60^{\circ} \mathrm{C}$.

2.3. Characterization. The microscopic morphologies of samples were characterized by transmission electron microscopy (TEM; JEOL inc., EM2100) and dynamic force microscope (DFM, Seiko Instruments Inc., SPA$300 \mathrm{HV})$. The structures of materials were measured by Fourier-transform infrared spectra analyzer (FT-IR; Thermo Scientific inc., NICOLET iS10) and X-ray diffraction (XRD; Rigaku inc., Ultima IV). UV spectrometer (UV-VIS; Hitachi inc., U-3900) was used to measure the optical absorption properties of GO. Thermo gravimetric analyzer (TGA; Perkin Elmer Inc., STA6000) was used to determine the thermal stability of samples. Elemental analyzer (EA; German Elementar Analysensysteme inc., D-63452 Hanau) and X-ray Photoelectron Spectroscope (XPS; England Thermo VGScientific inc., Sigma Probe) were used to analyze elementals in $\mathrm{GO}$ sheet.

\section{Results and Discussion}

3.1. The Morphologies of GO Sheet. Figure 1(a) showed the GO product prepared in the present study. It indicated that the GO sheet exhibited brown and very thin lamellar structure on the macrolevel, and the yellow performance was more significant in relatively low concentrations. Meanwhile, GO sheets prepared in this study were very slim and had certain toughness, not easy to grind. GO product was dispersed in anhydrous ethanol and supported on the copper mesh and Si chip for the TEM and DFM tests, respectively. The results are shown in Figures 1(b) and 1(c). Single and double lamellar layer structure GO sheets were observed in the TEM image, while the size of the lamellar structure was about 3 um and slight wrinkles were observed. The DFM image shows that the GO sheet layers have a thickness of $2 \sim 3 \mathrm{~nm}$, which was slightly thicker than single layer graphene. The increased thickness is largely attributed to the insertion of oxygencontaining functional groups which make it thicker and change the surface of the GO sheet. Moreover, since the oxygen-energy groups were mainly combined with edges part of GO sheets, resulting in the phenomenon that the thickness of GO sheets was high in the edge portion and low in the middle portion.

3.2. The Structures of GO Sheet. XRD analysis was used to determine the average crystalline properties of the GO sheet. Results are shown in Figure 2(a). The GO sheet prepared showed a very strong peak at $2 \theta=10.2^{\circ}$, which is in good agreement with the literatures $[23,24]$. The results of XRD initially proved the successful synthesis of GO sheet.

FTIR spectra analysis was performed to investigate the structure and functional groups of the materials, as shown in Figure 2(b). The GO sheet showed apparent adsorption bands for the carboxyl $\mathrm{C}=\mathrm{O}\left(1723 \mathrm{~cm}^{-1}\right)$, aromatic $\mathrm{C}=\mathrm{C}\left(1621 \mathrm{~cm}^{-1}\right)$, epoxy $\mathrm{C}-\mathrm{O}\left(1220 \mathrm{~cm}^{-1}\right)$, alkoxy $\mathrm{C}-\mathrm{O}$ $\left(1043 \mathrm{~cm}^{-1}\right)$, and hydroxy $-\mathrm{OH}\left(3391 \mathrm{~cm}^{-1}\right)$ groups. The presentation of oxygen-containing functional groups, such as $\mathrm{C}=\mathrm{O}$ and $\mathrm{C}-\mathrm{O}$, further confirmed that the graphite indeed was oxidized into GO and was consistent with the literatures [25-27]. The presentation of $\mathrm{C}=\mathrm{C}$ groups showed that even graphite had been oxidized into GO; the main structure of layer graphite was still retained. The results of XRD and FT-IR 


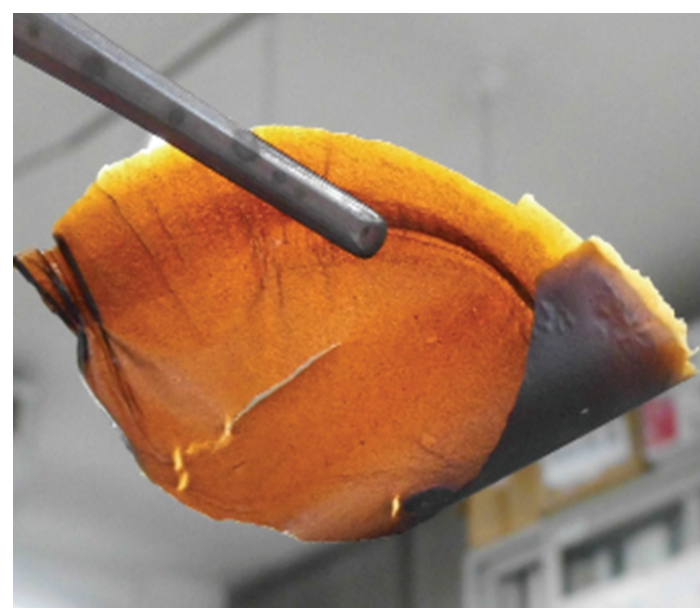

(a)

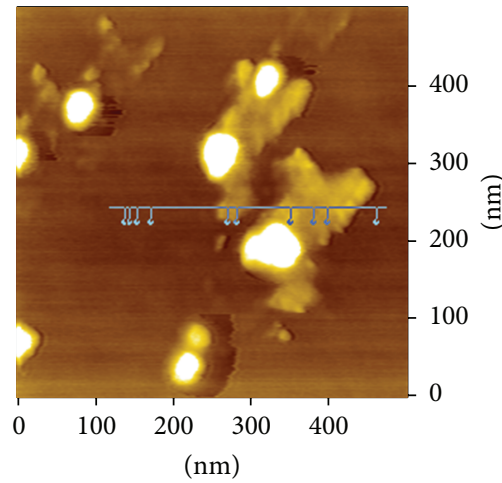

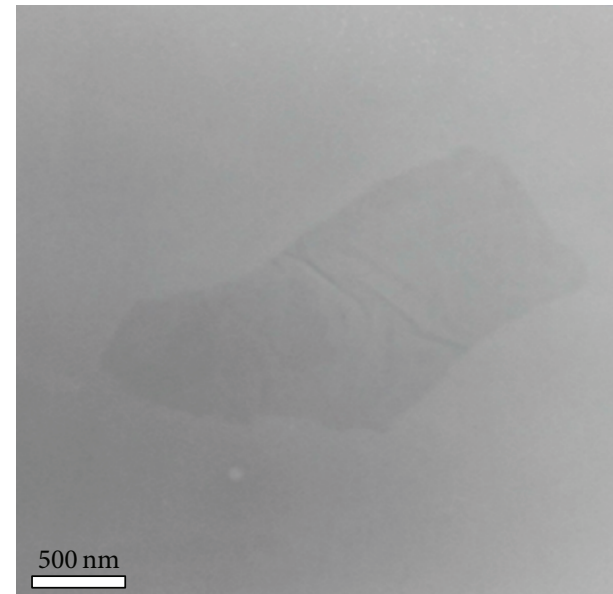

(b)

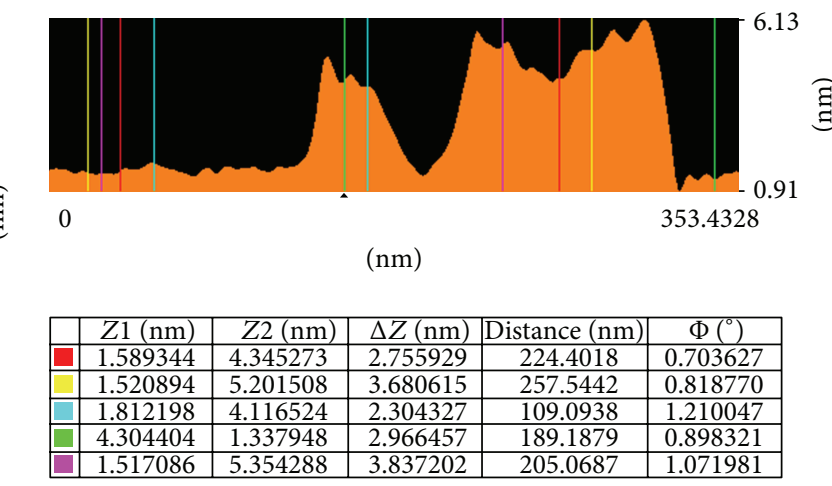

(c)

FIGURE 1: (a) The picture of GO product; (b) TEM; and (c) DFM pictures of GO sheet.

synthesis both further demonstrated the successful synthesis of GO sheet.

3.3. The Optical Absorption Properties of GO Sheet. The analysis of UV-VIS diffuse reflectance spectra of the GO sheet was shown in Figure 3(a). It is indicated that graphene oxide possessed a good absorption in the visible range $(380 \sim 800 \mathrm{~nm})$, but absorption in the ultraviolet range was also slightly decreased. The results showed the good photoresponse of GO sheet not only in ultraviolet range but also in visible range, which implied the enormous potential for application of light.

3.4. The Thermal Stability of GO Sheet. TGA analysis was conducted to test the thermal stability of GO sheet. Results were shown in Figure 3(b). Three stages were observed in the quality loss cure of the GO sheet. Firstly, a roughly $2 \%$ quality loss occurred at the temperature of $100^{\circ} \mathrm{C}$, primarily due to the loss of $\mathrm{H}_{2} \mathrm{O}$ molecules in the GO sheet layers. Secondly, the thermal decomposition of instable oxygen-containing functional groups showed a roughly $32 \%$ quality loss, occurring at a temperature of $225^{\circ} \mathrm{C}$. Finally, a $45 \%$ quality loss occurred at $620^{\circ} \mathrm{C}$ was mainly due to the combustion of the carbon skeleton. The analysis results indicated the excellent
TABLE 1: The EA results of GO sheet.

\begin{tabular}{lcccc}
\hline Element\% & $\mathrm{C} \%$ & $\mathrm{H} \%$ & $\mathrm{~S} \%$ & $\mathrm{~N} \%$ \\
\hline GO & 44.2 & 3.0 & 0.9 & 0.00 \\
\hline
\end{tabular}

thermal stability of GO sheet. Graphite oxide was very stable substance in the normal state.

3.5. The Elementals Synthesis of GO Sheet. During the process of preparing the GO sheet via the modified Hummer method, the added mass of pure graphite was $5.0 \mathrm{~g}$, and the final quantity of the obtained GO sheet was about $11.1 \mathrm{~g}$. From this, we can theoretically infer that the content of $\mathrm{C}$ element in GO sheet was about $45.0 \%$, which was consistent with the experimental results of the EA analysis, as shown in Table 1. In addition, $\mathrm{H}(3 \%)$ and $\mathrm{S}(0.9 \%)$ were observed in the GO sheet, primarily due to the presence of oxygen-containing functional groups embedded in the GO sheet. Meanwhile, based on the results of the EA, the $\mathrm{O}$ element content in the GO film was about $51 \%$.

3.6. The XPS Analysis of GO Sheet. In order to further demonstrate the presentation of oxygen-containing functional groups, XPS analysis was conducted. The result was 


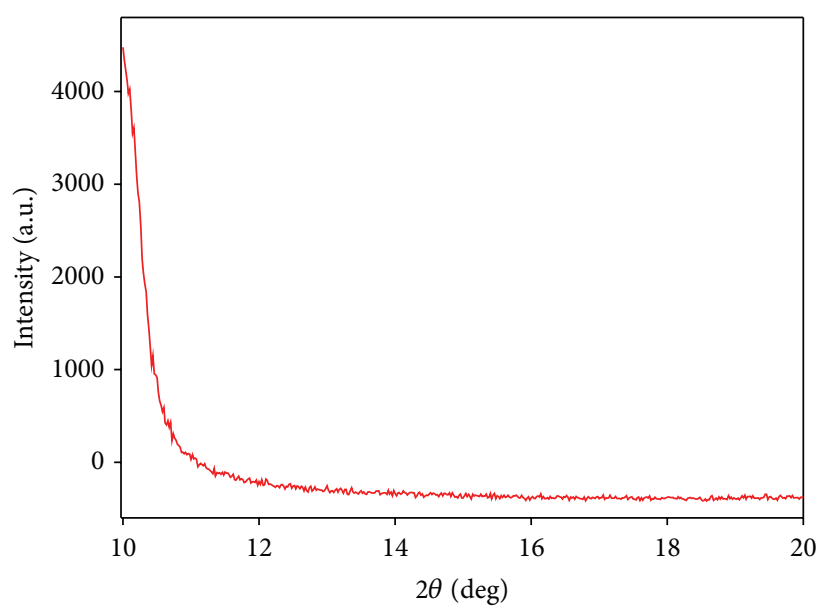

(a)

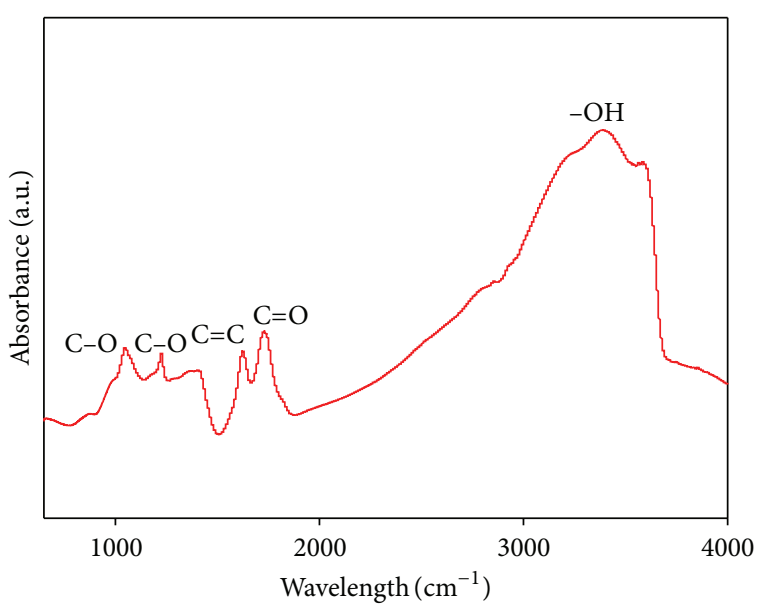

(b)

FIGURE 2: The (a) XRD and (b) FT-IR patters of GO sheet.

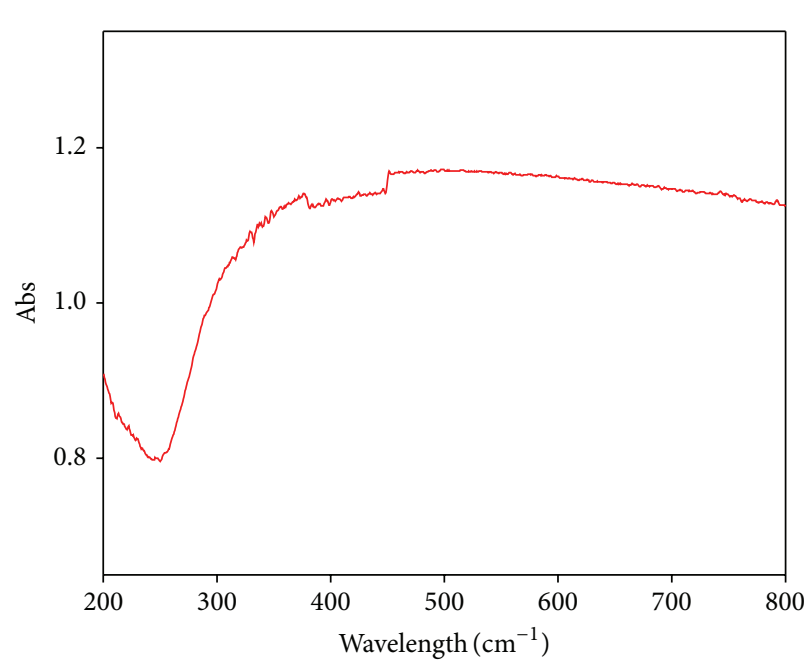

(a)

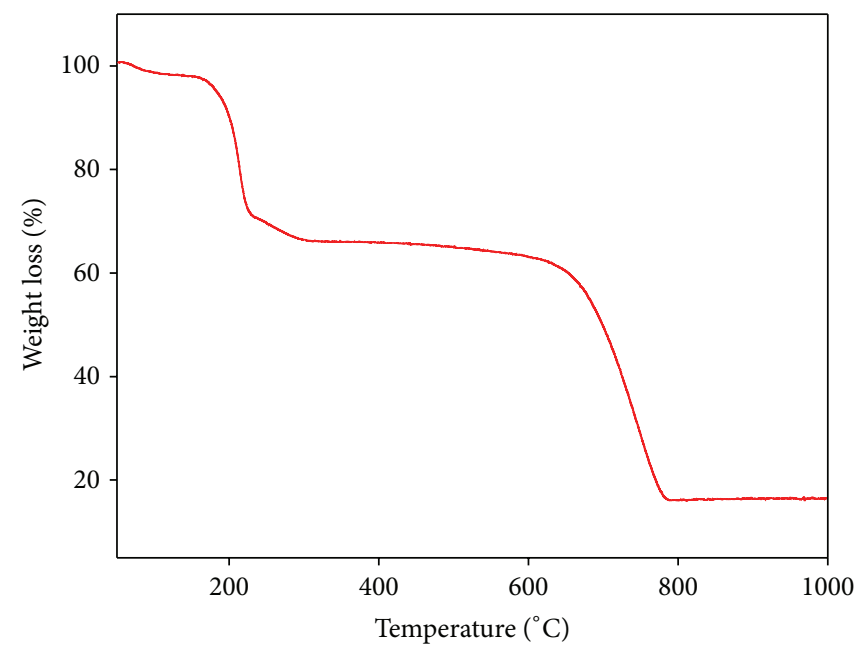

(b)

FIGURE 3: (a) The UV-VIS spectra of GO sheet; (b) the thermal gravimetric analysis diagram of GO sheet.

shown in Figure 4. XPS of GO showed significant $C$ and $\mathrm{O}$ signals corresponding to the binding energy of GO. Figure 4(a) showed the significant peak of $\mathrm{C}$ signal with a binding energy of $285.0 \mathrm{eV}$, which can be attributed to the $\mathrm{C}=\mathrm{C}, \mathrm{C}-\mathrm{C}$ and $\mathrm{C}-\mathrm{H}$ functional groups. The peak centered at the binding energies of $532.1 \mathrm{eV}$ can be assigned to the C$\mathrm{O}$ and $\mathrm{C}=\mathrm{O}$ bonds, as shown in Figure 4(b) [28]. This result suggested that $\mathrm{GO}$ sheet contains large numbers of functional groups on its surface, such as $\mathrm{C}-\mathrm{O}$ and $\mathrm{C}=\mathrm{O}$, which also was in accordance with the XRD, FTIR, and EA analyses.

3.7. Structural Analysis of Graphite Oxide (GO). Graphene oxide (GO) was a specific branch of graphene research. It can be considered as a precursor for graphene synthesis by either thermal or chemical reduction processes. The schematic structure of graphite oxide (GO) was shown in Figure 5. It is indicated that GO sheet can be regarded as the combined structure of oxygen-containing functional groups, such as $\mathrm{C}-$ $\mathrm{O}, \mathrm{C}=\mathrm{O}$, and $-\mathrm{OH}$, supported on the surface of a single layer graphene sheet $[7,29]$. The addition of oxygen-containing functional groups changed the structure and properties of GO sheet.

Compared with single-layer graphene, on the one hand, the presence of oxygen-containing functional groups indeed greatly increased defectiveness of GO sheet. This may lead to some loss in electrical conductivity [29], which possibly limits the direct application of GO in many areas. On the other hand, the presence of these oxygen-containing functional groups also provided potential advantages for using GO in numerous other applications. For example, the polar oxygen functional groups in GO sheet render it strongly hydrophilic, which gave GO good dispersibility in many solvents, particularly in water $[16,30-32]$. This also provided the conditions to synthesize composite materials. 


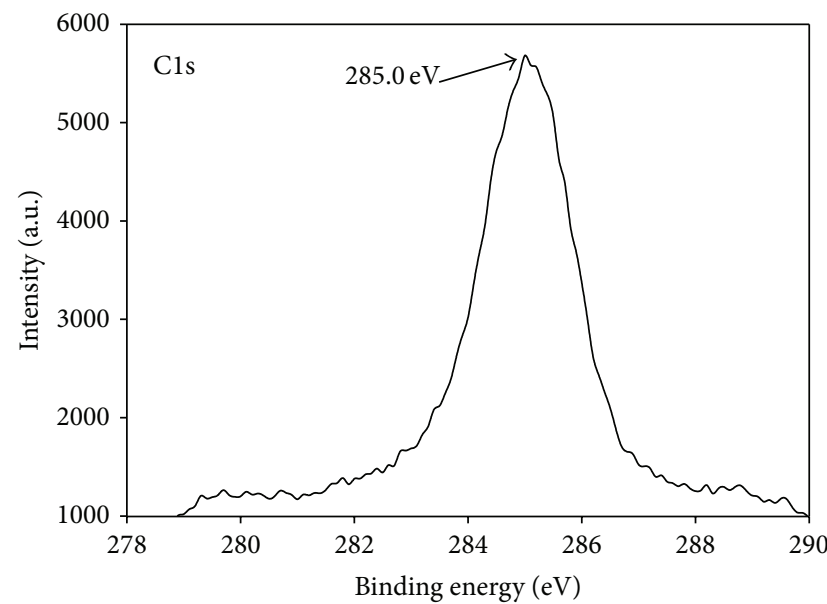

(a)

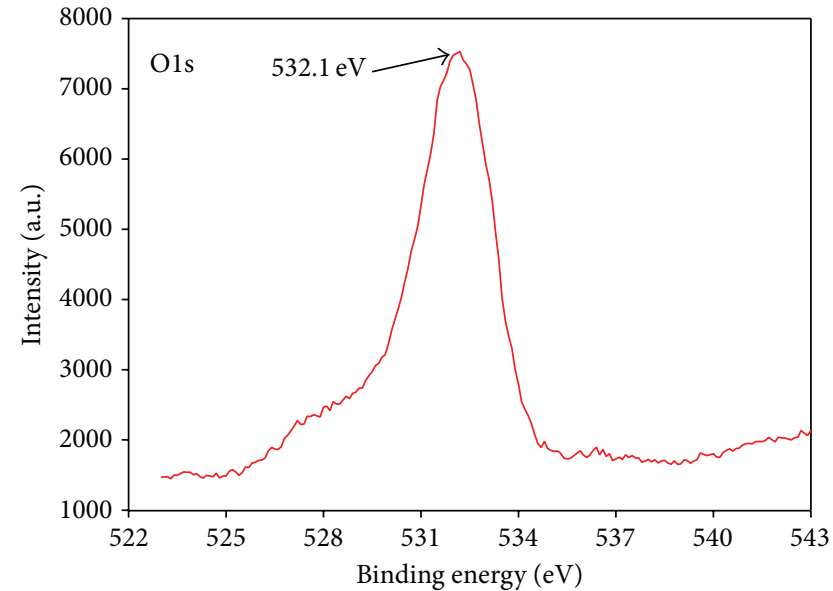

(b)

FIgURE 4: The C 1s (a) and O1s (b) core-level XPS spectra of GO sheet.

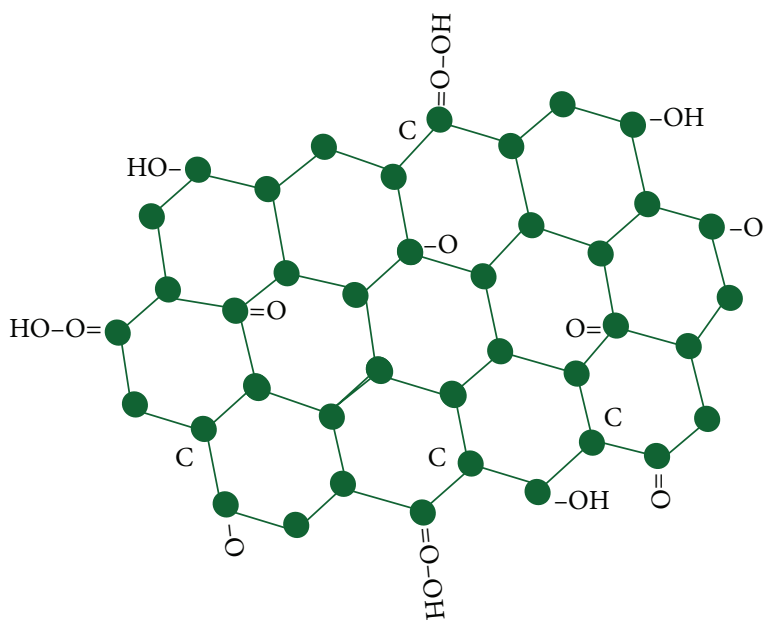

FIGURE 5: The schematic structure of graphite oxide (GO).

Simultaneously, these functional groups can be served as sites for chemical modification or functionalization of GO sheet, in order to easily apply in many other aspects.

\section{Conclusions}

GO films were successfully prepared via modified Hummer method. The TEM and DFM analyses showed successful preparation of GO films on microscopic morphology. The thickness of the monolayer GO sheet was between 2-3 nm.

The presence of oxygen-containing groups and characteristic peaks in FT-IR and XRD analysis further determined the successful preparation of GO sheets. Finally, EA indicated the component of $\mathrm{O}$ elements in GO films was about $51 \%$; elements of $\mathrm{C}, \mathrm{H}$, and $\mathrm{S}$ were also observed. The presence of oxygen-containing functional groups provided more opportunities for potential applications of GO in many areas. These data will provide a reference to further study the nature of graphene and graphene oxide.

\section{Conflict of Interests}

The authors declare that there is no conflict of interests regarding the publication of this paper.

\section{References}

[1] P. R. Wallace, "The band theory of graphite," Physical Review, vol. 71, no. 9, pp. 622-634, 1947.

[2] H. P. Boehm, A. Clauss, G. O. Fischer, and U. Hofmann, "Dünnste kohlenstoff-folien," Verlag der Zeitschrift für Naturforschung B, vol. 17, pp. 150-153, 1962.

[3] A. J. van Bommel, J. E. Crombeen, and A. van Tooren, "LEED and Auger electron observations of the $\mathrm{SiC}(0001)$ surface," Surface Science, vol. 48, no. 2, pp. 463-472, 1975.

[4] I. Forbeaux, J.-M. Themlin, and J.-M. Debever, "Heteroepitaxial graphite on $6 \mathrm{H}-\mathrm{SiC}(0001)$ : interface formation through conduction-band electronic structure," Physical Review B, vol. 58, no. 24, pp. 16396-16406, 1998.

[5] C. Oshima, A. Itoh, E. Rokuta, T. Tanaka, K. Yamashita, and T. Sakurai, "Hetero-epitaxial-double-atomic-layer system of monolayer graphene/monolayer $h$-BN on Ni(111)," Solid State Communications, vol. 116, no. 1, pp. 37-40, 2000.

[6] K. S. Novoselov, A. K. Geim, S. V. Morozov et al., "Electric field in atomically thin carbon films," Science, vol. 306, no. 5696, pp. 666-669, 2004.

[7] M. D. Stoller, S. Park, Z. Yanwu, J. An, and R. S. Ruoff, "Graphene-based ultracapacitors," Nano Letters, vol. 8, no. 10, pp. 3498-3502, 2008.

[8] X. Li, G. Zhang, X. Bai et al., "Highly conducting graphene sheets and Langmuir-Blodgett films," Nature Nanotechnology, vol. 3, no. 9, pp. 538-542, 2008.

[9] K. S. Novoselov, A. K. Geim, S. V. Morozov et al., "Twodimensional gas of massless Dirac fermions in graphene," Nature, vol. 438, no. 7065, pp. 197-200, 2005.

[10] K. S. Novoselov, Z. Jiang, Y. Zhang et al., "Room-temperature quantum hall effect in graphene," Science, vol. 315, no. 5817, p. 1379, 2007. 
[11] A. A. Balandin, S. Ghosh, W. Bao et al., "Superior thermal conductivity of single-layer graphene," Nano Letters, vol. 8, no. 3, pp. 902-907, 2008.

[12] S. Ghosh, I. Calizo, D. Teweldebrhan et al., "Extremely high thermal conductivity of graphene: prospects for thermal management applications in nanoelectronic circuits," Applied Physics Letters, vol. 92, no. 15, Article ID 151911, 2008.

[13] S. K. Srivastava, A. K. Shukla, V. D. Vankar, and V. Kumar, "Growth, structure and field emission characteristics of petal like carbon nano-structured thin films," Thin Solid Films, vol. 492, no. 1-2, pp. 124-130, 2005.

[14] C. E. Hamilton, J. R. Lomeda, Z. Sun, J. M. Tour, and A. R. Barron, "High-yield organic dispersions of unfunctionalized graphene," Nano Letters, vol. 9, no. 10, pp. 3460-3462, 2009.

[15] H. Jiang, "Chemical preparation of graphene-based nanomaterials and their applications in chemical and biological sensors," Small, vol. 7, no. 17, pp. 2413-2427, 2011.

[16] S. Park and R. S. Ruoff, "Chemical methods for the production of graphenes," Nature nanotechnology, vol. 4, no. 4, pp. 217-224, 2009.

[17] D. A. Dikin, S. Stankovich, E. J. Zimney et al., "Preparation and characterization of graphene oxide paper," Nature, vol. 448, no. 7152, pp. 457-460, 2007.

[18] W. S. Hummers Jr. and R. E. Offeman, "Preparation of graphitic oxide," Journal of the American Chemical Society, vol. 80, no. 6, p. 1339, 1958.

[19] S. Guo and S. Dong, "Graphene nanosheet: synthesis, molecular engineering, thin film, hybrids, and energy and analytical applications," Chemical Society Reviews, vol. 40, no. 5, pp. 26442672, 2011.

[20] C. Zhu, S. Guo, Y. Fang, and S. Dong, "Reducing sugar: new functional molecules for the green synthesis of graphene nanosheets," ACS Nano, vol. 4, no. 4, pp. 2429-2437, 2010.

[21] C. N. R. Rao, K. S. Subrahmanyam, H. S. S. Ramakrishna Matte, U. Maitra, K. Moses, and A. Govindaraj, "Graphene: synthesis, functionalization and properties," International Journal of Modern Physics B, vol. 25, no. 30, pp. 4107-4143, 2011.

[22] S. D. Perera, R. G. Mariano, K. Vu et al., "Hydrothermal synthesis of graphene- $\mathrm{TiO}_{2}$ nanotube composites with enhanced photocatalytic activity," ACS Catalysis, vol. 2, no. 6, pp. 949-956, 2012.

[23] D. C. Marcano, D. V. Kosynkin, J. M. Berlin et al., "Improved synthesis of graphene oxide," ACS Nano, vol. 4, no. 8, pp. 48064814, 2010.

[24] Z. J. Fan, K. Wang, T. Wei, J. Yan, L. P. Song, and B. Shao, "An environmentally friendly and efficient route for the reduction of graphene oxide by aluminum powder," Carbon, vol. 48, no. 5, pp. 1670-1692, 2010.

[25] K. Zhou, Y. Zhu, X. Yang, X. Jiang, and C. Li, "Preparation of graphene- $\mathrm{TiO}_{2}$ composites with enhanced photocatalytic activity," New Journal of Chemistry, vol. 35, no. 2, pp. 353-359, 2011.

[26] Y. Zhang and C. Pan, " $\mathrm{TiO}_{2}$ /graphene composite from thermal reaction of graphene oxide and its photocatalytic activity in visible light," Journal of Materials Science, vol. 46, no. 8, pp. 2622-2626, 2011.

[27] J. Shen, M. Shi, B. Yan, H. Ma, N. Li, and M. Ye, "Ionic liquidassisted one-step hydrothermal synthesis of $\mathrm{TiO}_{2}$-reduced graphene oxide composites," Nano Research, vol. 4, no. 8, pp. 795-806, 2011.
[28] D. Mo and D.-Q. Ye, "Surface study of composite photocatalyst based on plasma modified activated carbon fibers with $\mathrm{TiO}_{2}$," Surface and Coatings Technology, vol. 203, no. 9, pp. 1154-1160, 2009.

[29] D. R. Dreyer, S. Park, C. W. Bielawski, and R. S. Ruoff, "The chemistry of graphene oxide," Chemical Society reviews, vol. 39, no. 1, pp. 228-240, 2010.

[30] R. Bissessur and S. F. Scully, "Intercalation of solid polymer electrolytes into graphite oxide," Solid State Ionics, vol. 178, no. 11-12, pp. 877-882, 2007.

[31] J. I. Parades, S. Villar-Rodil, A. Martínez-Alonso, and J. M. D. Tascón, "Graphene oxide dispersions in organic solvents," Langmuir, vol. 24, no. 19, pp. 10560-10564, 2008.

[32] O. C. Compton and S. T. Nguyen, "Graphene oxide, highly reduced graphene oxide, and graphene: versatile building blocks for carbon-based materials," Small, vol. 6, no. 6, pp. 711$723,2010$. 

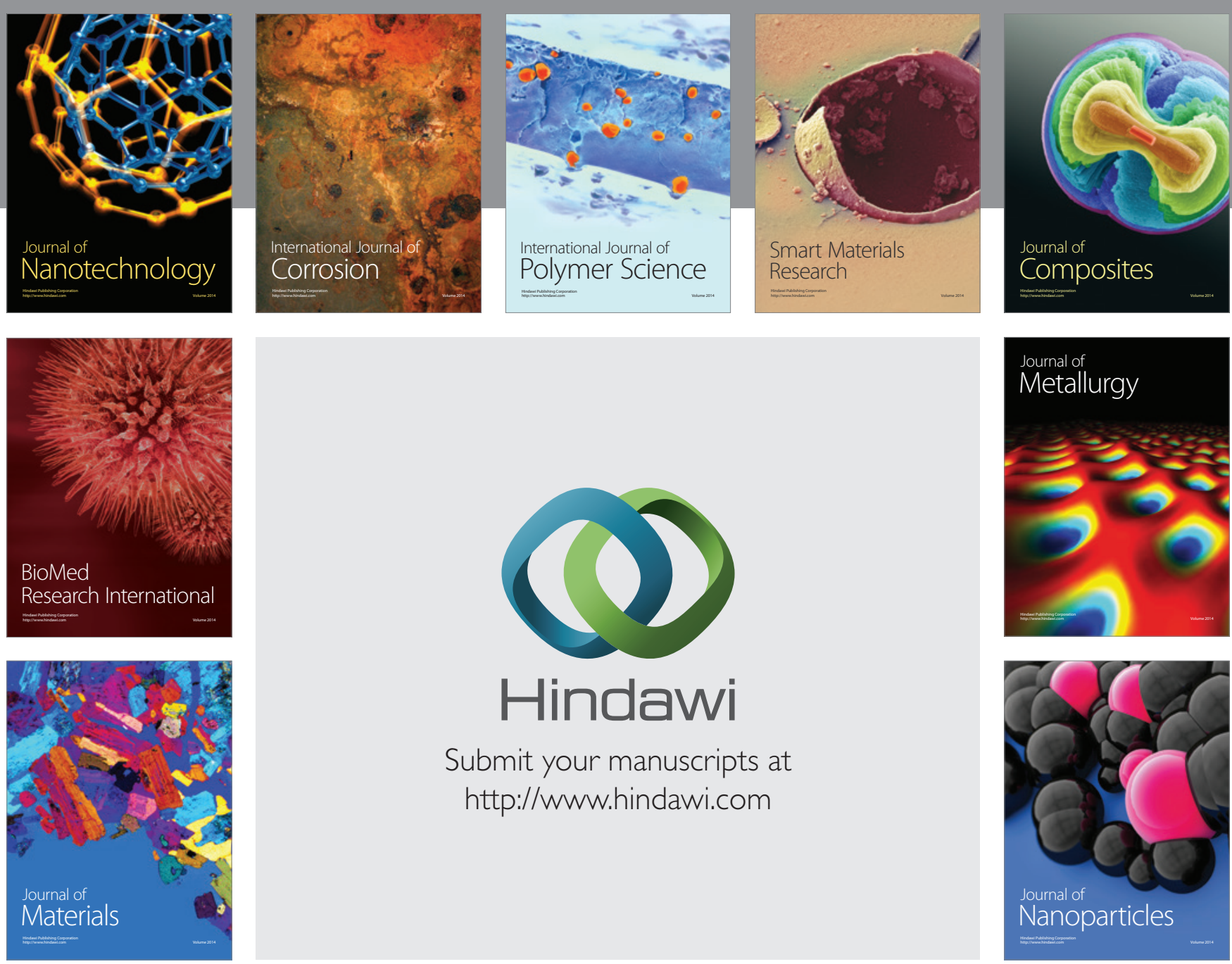

Submit your manuscripts at http://www.hindawi.com
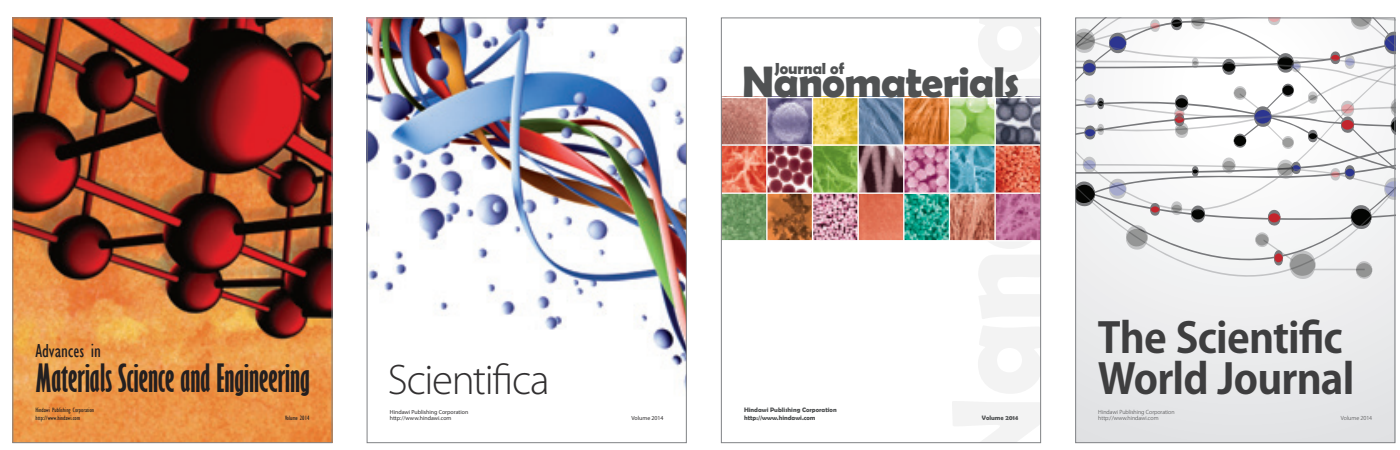

\section{The Scientific World Journal}
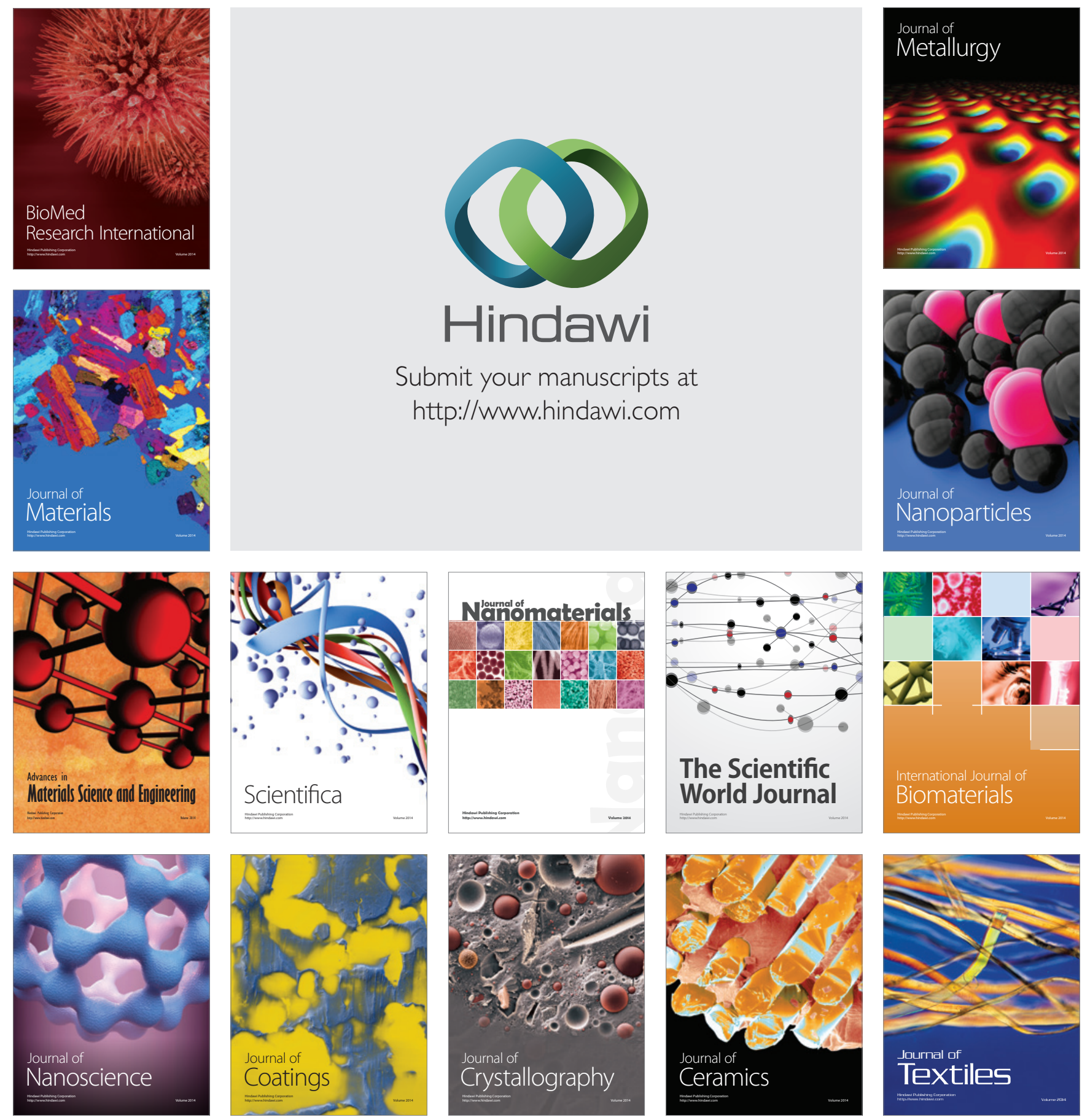